\title{
Über Molybdänverbindungen des Lecithins.
}

\section{Von}

R. Ehrenfeld.

\begin{abstract}
(Vorläufige Mitteilung aus dem Laboratorium des Prof. Dr. J. Habermann, k. k. technische Hochschule in Brünn.)

(Der Redaktion zugegangen am 26. April 1908.)
\end{abstract}

Von Verbindungen des Lecithins mit Schwermetallsalzen sind bisher nur die Platinchlorid- und die Cadmiumchloridverbindung beschrieben worden. ${ }^{1}$ ) Nebst dem theoretischen Interesse an den Metallsalzverbindungen des Lecithins weist auch noch ein praktisches Interesse auf das Studium dieser Verbindungen hin. Das Lecithin, frisch durch Alkoholextraktion des entölten Eidotters hergestellt, eine lichtgelbe wachsartige Masse, erleidet beim.Lagern an der Luft eine so intensive Zersetzung, daß es schließlich eine fast schwarze salbenartige Masse darstellt. Somit liegt die Frage offen, ob es nicht angängig ist, durch einen quantitativen Fällungsprozeß mit Hilfe eines Metallsalzes das unzersetzte Lecithinmolekül aus seinen alkoholischen Lösungen niederzuschlagen und dadurch neben seinen Zersetzungsprodukten analytisch zu bestimmen. Von dieser Erwägung geleitet wurden $3 \mathrm{~g}$ Lecithin (durch Alkoholextraktion aus vorher entöltem Eidotter gewonnen) in alkoholischer Lösung mit einigen Kubikzentimetern $1 / 10 \mathrm{n}$-Barytwasser bei stark alkalischer Reaktion am Rückflußkühler durch einige Zeit im Sieden erhalten, die Hauptmasse des Baryts durch einen Kohlensäurestrom, und die letzten Anteile durch genaue Fällung mittels $1 / 100$ n-Schwefelsäure entfernt. Hierauf wurde die Hauptmenge des Alkohols am Wasserbade verdunstet und zum Rückstande reichlich Wasser bis zur klaren, gelblich gefärbten Lösung hinzugefügt. Diese Auflösung der Komponenten des Lecithinmoleküls (Glycerinphosphorsäure, Fettsäuren, Cholin) wurde

1) Strecker, Annal. d. Chem., Bd. CXLVIII, S. 80. 
nun der Reihe nach mit einprozentigen wässerig-alkoholischen Lösungen fast aller Metallsalze (mit Ausnahme der Alkalimetalle und der seltenen Erden) auf Niederschläge geprüft, solche jedoch nur mit Hilfe des Cadmiumchlorids und des neutralen Bleiacetats gewonnen. Auch Phosphorwolframsäurelösungen liefern keinen Niederschlag. Parallel hierzu wurde eine frisch hergestellte alkoholische Lösung von $3 \mathrm{~g}$ Lecithin mit denselben Metallsalzlösungen und der Phosphorwolframsäurelösung geprüft und in der überwiegenden Zahl der Fälle reichliche Niederschläge erhalten, zu deren systematischer Durchforschung die Versuche bereits im Gange sind.

Im folgenden soll vorläufig nur über Lecithinverbindungen berichtet werden, welche mit Hilfe des Ammoniummolybdats erhalten wurden. In allen Fällen wurde eine Lösung von $3 \mathrm{~g}$ Lecithin in $200 \mathrm{ccm}$ absoluten Alkohols verwendet und die Fällung einerseits mit wechselnden Mengen der salpetersauren Lösung des Ammoniummolybdats, wie sie zur Fällung der Phosphorsäure Verwendung findet, ${ }^{1}$ ) vorgenommen, anderseits mit wechselnden Mengen der wässerigen Lösung des Ammoniummolybdats. Im ersteren Falle wurde die salpetersaure Lösung des Fällungsreagenzes mit der Hälfte des Volumens an absolutem Alkohol versetzt. Auf diese Art wurden drei verschiedene Verbindungen erhalten, die im folgenden unter I, II und III beschrieben werden sollen.

\section{I.}

Zur Lecithinlösung wurden das eine Mal $160 \mathrm{ccm}(\mathrm{A})$, das andere Mal bloß $80 \mathrm{ccm}$ (B) der salpetersauer-alkoholischen Lösung des Ammoniummolybdats in der Kälte hinzugefügt. In beiden Fällen entstanden, wie die Analyse es lehren sollte, identische Verbindungen in Form von krystallinischen, gelbgrünen Niederschlägen, die auf der Pumpe abgesaugt, mit verdünntem Alkohol (2 Teile absoluten Alkohols, und 1 Teil Wasser) genügend gewaschen und im Vakuum über Schwefelsäure zur Gewichtskonstanz getrocknet wurden. In diesen, wie in allen folgenden Fälle'n wurde der Stickstoff nach

1) $375 \mathrm{~g}$ Ammoniummolybdat in 2,5 1 Wasser gelöst und diese Lösung in 2,5 1 Salpetersäure (spez. Gew. $=1,2$ ) eingegossen. 
Kjeldahl bestimmt, und das Molybdän als $\mathrm{MoO}_{3}$ zur Wägung gebracht. $0,3 \mathrm{~g}$ der Verbindung wurden in alkoholischem Ammoniak in der Wärme gelöst und Schwefelwasserstoff bis zur dunklen Rotfärbung eingeleitet, wobei sich in einzelnen Fällen mit der fortschreitenden Bindung des Ammoniaks durch den Schwefelwasserstoff ein krystallinischer Molybdänsäureniederschlag ausschied, welcher nur durch einen neuerlichen, größeren Zusatz von Ammoniak wiederum in Lösung zu bringen war. Es war daher weit vorteilhafter, die Substanz in einen Porzellantiegel einzuwägen, und denselben in einen Nickeltiegel zu stellen, der zur dunklen Rotglut nach und nach erhitzt wurde. Der Lecithinanteil verascht in kürzester Zeit; der gelbliche, phosphorsäurehaltige Rückstand löst sich leicht in Ammoniak zu einer Lösung, die während des Einleitens von Schwefelwasserstoff völlig klar bleibt. Das durch Ansäuern der dunkelroten Lösung erhaltene, braune Molybdänsulfid wurde über dem Goochtiegel gesammelt und nach genügendem Waschen mittels Wasser und Alkohol weiter so behandelt, wie es Treadwell in seinem «Kurzen Lehrbuch der Analytischen Chemie», Bd. II, S. 184 angibt. Das durch Verglühen des Molybdänsulfids erhaltene Molybdäntrioxyd ist vielfach von glitzernden Krystallblättchen durchsetzt und hat einen Stich ins Bläuliche. Gewichtskonstanz war in allen Fällen dann erreicht, wenn der Geruch nach Schwefeldioxyd verschwunden und darüber hinaus noch einige Zeit geglüht worden war. Jede der beiden Verbindungen (A) und (B) war zweimal separat hergestellt und für sich analysiert worden. Die Resultate, aus mindestens zwei befriedigend übereinstimmenden Analysen im Mittel berechnet, sind die folgenden: 
Von Fettsäuren sind bekanntlich bisher Palmitin-, Stearin-, Ölsäure und schließlich auch Leinölsäure ${ }^{1}$ ) im Ovolecithin aufgefunden worden. Wird der Berechnung die Formel:

$$
10 \mathrm{MoO}_{3} \cdot \overbrace{\text { Dioleinlecithin }}^{3 \mathrm{C}_{44} \mathrm{H}_{86} \mathrm{O}_{9} \mathrm{NP}}
$$

zugrunde gelegt, dann resultieren bis auf Unterschiede in den Zehntelperzent dieselben Perzentzahlen für $\mathrm{MoO}_{3}$ und $\mathrm{N}$ wie bei der Verbindung $10 \mathrm{MoO}_{3} \cdot 3 \mathrm{C}_{44} \mathrm{H}_{90} \mathrm{O}_{9} \mathrm{NP}$. Die gefundenen Perzentzahlen für $\mathrm{MoO}_{3}$ liegen ganz folgerichtig zwischen den berechneten Zahlen für: $10 \mathrm{MoO}_{3} \cdot 3 \mathrm{Mol}$. Distearolecithin und $10 \mathrm{MoO}_{3} \cdot 3 \mathrm{Mol}$. Dipalmitolecithin.

\section{II.}

Eine zweite Verbindung wurde so hergestellt, daß zur alkoholischen Lecithinlösung bloß $17 \mathrm{ccm}$ der salpetersauer-alkoholischen Reagenzlösung hinzugefügt wurde, so daß das Lecithin in großem Überschusse vorhanden war. Es resultiert ein gelblichgrüner, krystallinischer Niederschlag in der Kälte, der mittels absoluten Alkohols lecithinfrei gewaschen und im Vakuum über Schwefelsäure zur Gewichtskonstanz getrocknet wird. Die Analysen von zwei separat dargestellten Mengen ergeben folgende Mittelwerte :

$$
\begin{array}{cr}
\text { Gefunden: } \\
\mathrm{MoO}_{3}: 27,52 \% \\
\mathrm{~N}: & 1,28 \%
\end{array}\left\{\begin{array}{c}
\text { Berechnet für } 2 \mathrm{MoO}_{8} \cdot\left(\mathrm{C}_{40} \mathrm{H}_{82} \mathrm{O}_{0} \mathrm{NP}\right) \\
\mathrm{MoO}_{3}: 27,70 \% . \\
\mathrm{N}: \quad 1,34 \% . \\
\text { Berechnet für } 2 \mathrm{MoO}_{8} \cdot\left(\mathrm{C}_{44} \mathrm{H}_{80} \mathrm{O}_{9} \mathrm{NP}\right) \\
\mathrm{MoO}_{3}: 26,28 \% . \\
\mathrm{N}: \quad 1,27 \% .
\end{array}\right.
$$

Beide Verbindungen: $10 \mathrm{MoO}_{3} \cdot 3 \mathrm{Mol}$. Lecithin sowie $2 \mathrm{MoO}_{3} \cdot 1 \mathrm{Mol}$. Lecithin liefern, in Wasser suspendiert und mit Magnesiumoxyd gekocht, kein Ammoniak. Ihrer Verwertbarkeit zur quantitativen Abscheidung des Lecithins steht die immerhin nicht unbeträchtliche Löslichkeit in wässerigem Alkohol im Wege. Schon beim Waschen dieser Verbindungen über der Pumpe mit einem Gemisch von zwei Teilen absoluten Alkohols mit einem Teil Wasser lieferte die Waschflüssigkeit beständig einen $A b-$

1) Cousin, Comptes rendus 1903, S. 68. 
dampfrückstand, der, mit einem Tropfen konzentrierter Schwefelsäure und Alkohol erwärmt, die blaue Farbe des Molybdänoxyds lieferte. Schon beim Schütteln mit diesem so verdünnten Alkohol entsteht eine opalisierende, grünlich gefärbte Lösung, die beim Kochen am Rückflußkühler mit schön grüner Farbe sich klärt. Noch leichter entsteht die grüne opalisierende Lösung beim Schütteln mit organischen Lösungsmitteln wie Benzol, Petroläther, Chloroform, Tetrachlorkohlenstoff $u$. ä.

III.

Schließlich wurde versucht, die alkoholische Lecithinlösung mit rein wässerigen Lösungen des Ammonmolybdats zu fällen. Wird zu einer Auflösung von $5 \mathrm{~g}$ Ammonmolybdat in $100 \mathrm{ccm}$ Wasser die Hälfte des Volumens an absolutem Alkohol hinzugefügt, dann scheidet sich der größte Teil des gelösten Stoffes aus. Daher wurde die Fällung so vorgenommen, daß die wässerige Ammonmolybdatlösung zur alkoholischen Lecithinlösung tropfenweise und unter Umschütteln hinzugebracht wurde, wobei das Lecithin stets im großen Überschuß vorhanden sein mußte. In einem Falle wurde zur alkoholischen Lecithinlösung eine Auflösung von $4 \mathrm{~g}$ Ammonmolybdat in $30 \mathrm{ccm}$ Wasser, im zweiten Falle eine solche von $14 \mathrm{~g}$ Ammonmolybdat in $100 \mathrm{ccm}$ Wasser in der Art hinzugefügt. Die entstehenden Produkte waren in beiden Fällen identisch und stellten rein weiße, krystallinische Niederschläge dar. Über der Pumpe mittels absoluten Alkohols lecithinfrei gewaschen und im Vakuum über Schwef elsäure zur Gewichtskonstanz getrocknet, lieferten sie im Mittel folgende Analysenresultate $:^{1}$ )

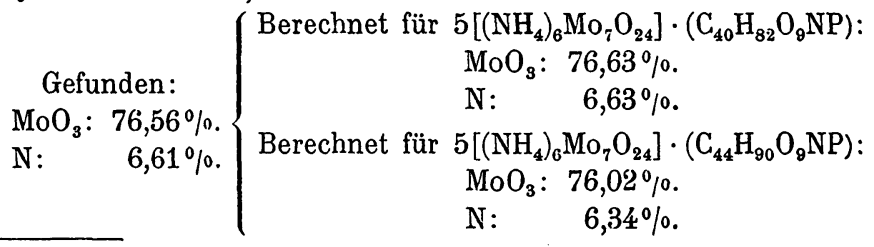

1) Es mag erwähnt werden, daß auch versucht wurde, den Veraschungsrückstand der Verbindungen in Ammoniak zu lösen, mit Salzsäure zu übersättigen und das Molybdän titrimetrisch nach der Methode von v. d. Pfordten (Berichte d. Deutschen chem. Gesellsch., Bd. XV, S. 1925) mittels Kaliumpermanganat zu bestimmen, wobei stets viel zu niedrige Resultate erhalten wurden, worüber noch Versuche im Gange sind. 
94 R. Ehrenfeld, Über Molybdänverbindungen des Lecithins.

Diese Verbindung löst sich im Wasser beträchtlich auf. Schon beim ruhigen Stehen resultiert eine gelb gefärbte Lösung, die sich auf Zusatz von Alkohol trübt.

\section{Zusammenfassung :}

1. Durch Fällen alkoholischer Lecithinlösungen mittels salpetersauer-alkoholischer Ammonmolybdatlösungen in der Kälte entstehen, je nach den stöchiometrischen Verhältnissen der aufeinander wirkenden Stoffe, die beiden Verbindungen:

$$
\begin{array}{r}
10 \mathrm{MoO}_{3} \cdot 3 \mathrm{Mol} \text {. Lecithin, } \\
2 \mathrm{MoO}_{3} \cdot 1 \mathrm{Mol} \text {. Lecithin. }
\end{array}
$$

Im ersteren Falle ist das Ammonmolybdat, im letzteren das Lecithin im Überschusse vorhanden.

2. Durch Fällen alkoholischer Lecithinlösungen mittels wässeriger Ammonmolybdatlösung in der Kälte, wobei das Lecithin stets im großen Überschuß vorhanden sein muß, entsteht die Verbindung:

$$
5\left[\left(\mathrm{NH}_{4}\right)_{6} \mathrm{Mo}_{7} \mathrm{O}_{24}\right] \cdot 1 \mathrm{Mol} \text {. Lecithin. }
$$

Für die quantitative Abscheidung des Lecithins kommen bloß die unter 1. angeführten Verbindungen in Betracht. Sie sind jedoch hierzu infolge ihrer Löslichkeit in wässerigem Alkohol ungeeignet. 